\title{
AN EXPERIMENTAL TEST OF THE RIGID-MUFFIN-TIN APPROXIMATION USED IN THE THEORY OF ELECTRON-PHONON INTERACTION
}

W. Ruesink, J. de Wilde, R. Griessen and M.J.G. Lee

Natuurkundig Laboratorium der Vrije Universiteit, Amsterdom, The Netheriands

Physics Department, University of Toronto, Toronto, Canada.

Résumé.- La validitê de 1'approximation du potentiel rigide est analysée à 1'aide de mesures de $1^{\prime}$ influence d'un cisaillemenț pur sur la surface de Fermi du palladium. Les valeurs expérimentales sont environ $30 \%$ plus petites que les valeurs obtenues à l'aide de la méthode KKR. Quelques implications relatives au couplage electron-phonon et à $T_{c}$ sont présentées.

Abstract. - The validity of the rigid-muffin-tin approximation is discussed on the basis of measur rements of the response of the Fermi surface of palladium to homogeneous volume conserving strains. The experimental strain derivatives are found to be consistently $30 \%$ smaller than theoretical values obtained from a KKR band structure calculations. Implications of this result are discussed for the electron-phonon $\lambda$ and the superconducting $T_{c}$.

According to the McMillan theory $/ 1,2 /$ the superconducting transition temperature $\mathrm{T}_{\mathrm{c}}$ of a metal depends on the electron-phonon enhancement factor $\lambda$ through the following relation

$\mathrm{T}_{c}=(1.2)^{-1} \omega_{10 g} \exp \left\{-1.04(1+\lambda) / \lambda-\mu^{\mathrm{x}}(1+0.62 \lambda)\right\}$

where $\mu^{\mathrm{x}}$ is a constant (assumed to be 0.13 ) and $\omega_{10 g}$ is an average over the phonon frequencies. The parameter $\lambda$ can be expressed as a product of a purely electronic factor $\eta$ and an essentially phononic factor so that : $\lambda=n / M<\omega^{2}>$

Here $M$ is the mass of the ions ; $\left\langle\omega^{2}\right\rangle$ depends only weakly on the electronic properties and is determined by the phonon spectrum of the metal under consideration. The central problem is the calculation of $\eta$ which is proportional/1/ to the gradient of the potential $\mathrm{V}$. Most of the recent theoretical work on electron-phonon interaction in transition metals makes use of the rigid-muffin-tin (RMT) approximation of Gaspari and Gyorffy /3/. In this approximation one assumes that the self-consistent muffintin potential around an ion moves rigidly with the ion when a phonon is propagating through the lattice. The validity of this approximation is difficult to test by means of superconducting $T_{c}$ data, because of the uncertainties in $\omega_{\log },\left\langle\omega^{2}\right\rangle$ and $\mu^{x}$. In this paper we present a direct method to test the validity of the RMT approximation based on measurements of the strain response of the Fermi surface (FS) of transition metals to homogeneous volume conserving strains (shears).

In order to show which role the RMT approximation plays in the shear response of the FS of a metal we write the KKR secular equation as $X\left(\vec{k}, E, \delta_{\ell}, e_{j}\right)=0$

where $\delta_{\ell}$ are scattering phase shifts of the muffintin potential and $e_{j}$ is the $j$-th component of the strain tensor. The strain dependence of the area $A$ of an extremal cross-section of the FS is obtained by solving the implicit equation (3) with respect to $\vec{k}$ at an energy equal to $E_{F}$. As shown by Shaw, Ketterson and Windmiller /4/ and by Griessen, Stanley and Lee $15 /$, the strain derivative $D_{j}=$ $\mathrm{d} l \mathrm{nA} / \mathrm{de}_{\mathbf{j}}$ can be calculated from an integral along the orbit containing the term $\mathrm{DX} / \mathrm{De}{ }_{j}$, where $\mathrm{DX} / \mathrm{De}{ }_{j}$ $=\frac{\partial X}{\partial E} \frac{d E_{F}}{d e_{j}}+\Sigma_{\ell} \frac{\partial X}{\partial \delta_{\ell}} \frac{d \delta_{\ell}}{d e_{j}}+\frac{\partial X}{\partial e_{j}}$

For a general strain $\xi$, the derivative $d \ln A / d \xi$ depends explicitly on the strain dependence of $E_{F}$ and the scattering phase shifts. For volume conserving strains $\gamma$ it is however easy to show that in a cubic crystal :

$\mathrm{d} \ln A / \mathrm{d} \gamma=\sum_{j=1}^{6} \partial \ln \mathrm{\operatorname {ln }} /\left.\partial \mathrm{e}_{\mathrm{j}}\right|_{\delta_{\ell}, \mathrm{E}_{\mathrm{F}}} \mathrm{de} \mathrm{j} / \mathrm{d} \gamma$

where $\mathrm{de}_{\mathbf{j}} / \mathrm{d} \gamma$ is entirely determined by the strain state of the crystal Eq. (5) shows explicitly that the response of the FS to a shear depends on the pertubation of the electronic band structure by the lattice deformation at constant values of $\delta_{\ell}$ and $E_{F}$. Therefore the shear response can be calculated from a KKR model using zero-pressure values for $E_{F}$ and $\delta_{\ell}$ and the validity of the RMT-approximation can be tested by comparing measured $d \ln A / d \gamma$ with calculated derivatives. Experimental values for $D_{j}$ were obtai- 


\section{C6-1098}

ned by simultaneously measuring the amplitude of quantum oscillations in the magnetization $(\tilde{M})$ and the sound velocity $\left(\tilde{v}_{s}\right)$. As shown by Testardi and Condon/7/ the strain dependence of the FS can be calculated from :

$$
\left\{D_{i} D_{j}\right\}=\tilde{v}_{s} C_{e f f} / \pi F M
$$

where $\mathrm{C}_{\text {eff }}$ is the effective elastic constant corresponding to the ultrasonic configuration, $F$ is the de Haas-van Alphen frequency and $\left\{\mathrm{D}_{i} \mathrm{D}_{j}\right\}$ is a combination of derivatives $D_{j}$.

The measurements were done on palladium single crystals of $4 \mathrm{~N}$ purity in magnetic fields up to 6.5 tesla. The oscillations in $M$ were detected by means of a fluxgate probe coupled to a superconducting flux transformer $/ 8,9 /$ and the oscillations in $\mathrm{v}_{\mathrm{s}}$ were measured with a continuous wave set-up. From the $D_{j}$ measured in several ultrasonic configurations we determined the response to tetragonal shear $\gamma_{z}\left(e_{1}=e_{2}=-\frac{1}{2} e_{3}\right)$ and angular shear $\gamma_{x y}$ of the hole-ellipsoids at point $X$ and $L$ of the Brillouin zone, listed in table I.
The experimental numbers are found to be $30 \%$ smaller than the theoritical values from the relativistic KKR calculation. This is also true for the tetragonal shear derivative of the be11y orbit $\Gamma|100|$ determined from the uniaxial stress measurements of Joss and Van der Mark /6/. This information may be used to estimate the errror introduced in $n$ by the use of the RMT approximation as in the deformation potential approximation $/ 10 / \eta$ is proportional to $(\mathrm{DX} / \mathrm{D} \xi)^{2}$, whereas $\mathrm{d} / \mathrm{nA} / \mathrm{d} \gamma$ is $/ 5 /$ proportional to $\mathrm{DX} / \mathrm{D} \xi$. As a consequence of this quadratic dependence we expect the RMT approximation to give values approximately 1.7 too high for $\eta$. This tendency has also been found by Butler /11/ in $\mathrm{Y}, \mathrm{Zr}, \mathrm{Tc}$ and $\mathrm{Rh}$, while Gyorffy /12/ pointed out that the experimental value of $n$ from measurements of $T_{c}$ in niobium is about 1.6 times lower than the calculated value of Butler /11/. It is interesting to point out that the $T_{c}$ of palladium obtained with a "scaled" value of ${ }^{c}=\eta_{\mathrm{RMT}} / 1.7$ is essentially zero $\left(\mathrm{T}_{\mathrm{c}} \sim 10^{-18} \mathrm{mK}\right)$ while the value calculated by Butler /11/ with the RMT leads to $5.5 \mathrm{mk}$.

TABLE I

Experimental and theoretical shearderivatives of the area of extremal cross-sections of the FS of palladium. $\gamma_{z}$ is a tetragonal shear in the $|001|$ direction and $\gamma_{x y}$ is equal to the decrease of the angle between $|100|$ and $|010|$ axes of the real space lattice induced by the shear. The parameters entering the KKR bandstructure calculations are :

$$
\begin{aligned}
& E_{F}=0.4816 \mathrm{a} . \mathrm{u} ., \delta_{0}=-0.09887 \mathrm{rad}, \delta_{1,1 / 2}=-0.00205 \mathrm{rad}, \\
& \delta_{1,3 / 2}=-0.04170 \mathrm{rad}, \delta_{2,3 / 2}=-0.23918 \mathrm{rad}, \delta_{2,5 / 2}=-0.29095 \mathrm{rad}
\end{aligned}
$$

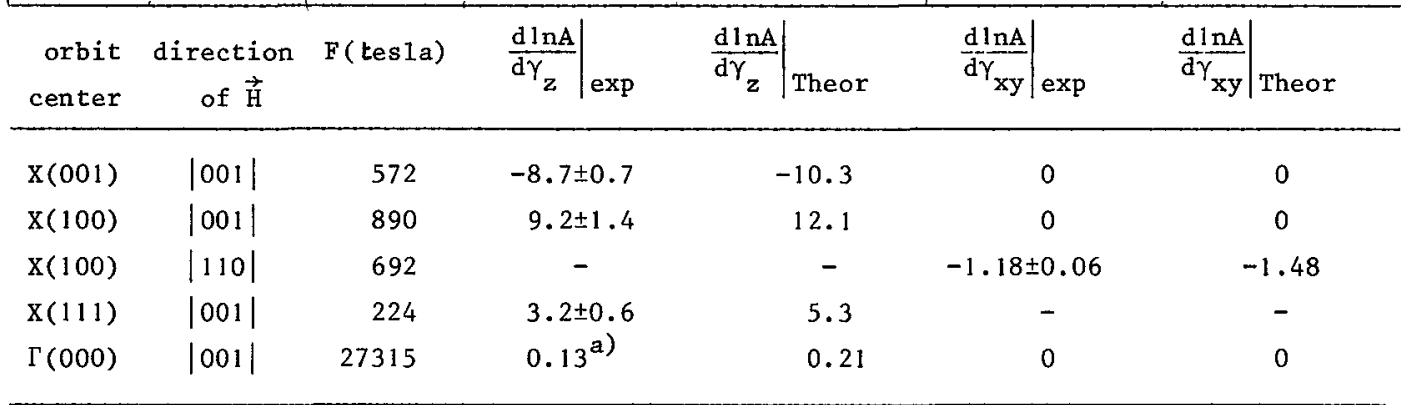

a) Obtained from uniaxial stress data $/ 6 /$

\section{References}

/1/ McMil1an, W.L., Phys. Rev. 167 (1968) 331

/2/ Allen, P.B. and Dynes, R.C., Phys. Rev. B12 (1975) 905

/3/ Gaspari, G.D. and Gyorffy, B.L., Phys. Rev. Lett. 28 (1972) 801

14/ Shaw, J.C., Ketterson, J.B. and Windmiller, L.R., Phys. Rev. B5 (1972) 3894

/5/ Griessen, R., Lee, M.J.G. and Stanley, D.J., Phys Rev. B16 (1977) 4385

/6/ Joss, W. and Van Der Mark, W., Proc. Int. Conf. on Physics of Transition Metals, Toronto 1977
17/ Testardi, L.R. and Condo, J.H. Phys. Rev. B1 (1970) 3928

/8/ De Wilde, J., Ph.D. Thesis (1978),Vrije Universiteit, Amsterdam

/9/ De Wilde, J. and Meredith, D.J., J. Phys. E9 (1976) 62

/10/ Ziman, J.M. "Electrons and Phonons" (Clarendon Press Oxford) 1962, p. 186

/11/ Butler, W.H., Phys. Rev. B15 (1977) 5267

/12/ Gyorffy, B.L. "Superconductivity in d- and fmetals" (Plenum Press, Ed. D.H. Douglass) 1972, p. 29 